\title{
UGC 12732: A DISK GALAXY LACKING HIGH-VELOCITY CLOUDS
}

\author{
ERIC SCHULMAN ${ }^{1}$ \\ National Radio Astronomy Observatory, 520 Edgemont Road, Charlottesville, Virginia 22903-2475 \\ Electronic mail: eschulma@nrao.edu \\ ELIAS BRINKS ${ }^{2}$ \\ National Radio Astronomy Observatory, P.O. Box O, Socorro, New Mexico 87801-0387 \\ Electronic mail: ebrinks@nrao.edu \\ JOEL N. BREGMAN \\ Department of Astronomy, University of Michigan, Ann Arbor, Michigan 48109-1090 \\ Electronic mail: jbregman@umich.edu \\ MORTON S. ROBERTS \\ National Radio Astronomy Observatory, 520 Edgemont Road, Charlottesville, Virginia 22903-2475 \\ Electronic mail: mroberts@nrao.edu \\ Received 1996 November 15; revised 1997 January 30
}

\begin{abstract}
We used the Very Large Array to obtain high-sensitivity $21 \mathrm{~cm}$ synthesis images of the disk galaxy UGC 12732. This object did not show high-velocity wings in Arecibo telescope spectra obtained to search for populations of high-velocity clouds in external galaxies. The VLA observations confirm the absence of high-velocity H I in UGC 12732: we set an upper limit of $2 \times 10^{7} \mathrm{M}_{\odot}$ for high-velocity material, consistent with the upper limit of $5 \times 10^{7} M_{\odot}$ inferred from the Arecibo observations. The gas distribution perpendicular to the disk is successfully represented by a thin layer with a constant Gaussian velocity dispersion of $\sigma_{v}=9 \mathrm{~km} \mathrm{~s}^{-1}$. UGC 12732 has a low star formation rate, which is consistent with previous findings that galaxies without active star formation lack high-velocity H I clouds. (C) 1997 American Astronomical Society. [S0004-6256(97)02205-X]
\end{abstract}

\section{INTRODUCTION}

The high-velocity clouds of neutral hydrogen in our Galaxy dominate the kinetic energy of $\mathrm{H} \mathrm{I}$ in non-circular motion and are an important though poorly understood component of Galactic gas. High-velocity clouds (HVCs) with $\left|v_{\text {LSR }}\right|>100 \mathrm{~km} \mathrm{~s}^{-1}$ cover approximately $18 \%$ of the sky to a limiting column density of 2 to $3 \times 10^{18} \mathrm{~cm}^{-2}$ (Wakker 1991). The nature and origin of these HVCs are not well understood, in part because the uncertain distances to the clouds prevent accurate determinations of their masses, linear diameters, or densities. Wakker (1990) showed that it is possible to reproduce the observed distribution of most of the Galactic high-velocity clouds with two populations of gas: galactic fountain gas from superbubbles that breaks out of the Galactic disk, cools radiatively as it rises upward, and eventually recombines and returns to the disk ballistically (Shapiro \& Field 1976; Bregman 1980), and Magellanic Stream material that is falling onto the Galaxy from the Magellanic Clouds (Murai \& Fujimoto 1980). High-velocity clouds in the galactic fountain model have a characteristic distance of 10 to $20 \mathrm{kpc}$ above the Galactic plane, while

\footnotetext{
${ }^{1}$ Jansky Fellow.

${ }^{2}$ Current address: Departamento de Astronomía, Universidad de Guanajuato, apartado Postal 144, Guanajuato, Gto. 36000, Mexico.
}

Magellanic Stream material has a characteristic distance of about $50 \mathrm{kpc}$ (Wakker 1990). If these two processes are the dominant sources for Galactic HVCs, then high-velocity clouds are a disk-wide phenomenon that comprises about $10 \%$ of the neutral hydrogen in the Galaxy. However, if most high-velocity clouds are local then there may be relatively little mass in HVCs. To date there has been only one distance determination to a high-velocity cloud: HVC Complex $\mathrm{M}$ is at a distance of $1.7<D<5.0 \mathrm{kpc}$ (Danly et al. 1993). Lower limits to the distance of several other high-velocity cloud complexes have been reported: HVC Complex $\mathrm{H}$ has $D>1.5 \mathrm{kpc}$ (Centurión et al. 1994), HVC Complex A has $D>4$ (Wakker et al. 1996a), and HVC Complex C has $D>2.5 \mathrm{kpc}$ (Wakker et al. 1996b). Because the distances almost all the HVCs in our Galaxy are unknown, further insight into the nature of high-velocity clouds can be obtained by finding and studying high-velocity gas in other galaxies and by investigating the differences between galaxies with and without HVCs.

A $21 \mathrm{~cm}$ survey of 14 nearly face-on disk galaxies using the Arecibo telescope ${ }^{3}$ revealed that $70 \%$ of the galaxies have high-velocity wings beyond the double-horned $\mathrm{H}$ I pro-

\footnotetext{
${ }^{3}$ The Arecibo Observatory is part of the National Astronomy and Ionosphere Center, which is operated by Cornell University under a cooperative agreement with the National Science Foundation.
} 
TABLE 1. General information on UGC 12732.

\begin{tabular}{|c|c|c|}
\hline Parameter & Value & Source \\
\hline Morphological type & Sm: & Nilson (1973) \\
\hline$\alpha$ (B1950) of optical center & $23^{\mathrm{h}} 38^{\mathrm{m}} 9^{\mathrm{s}} 1$ & Dressel \& Condon (1976) \\
\hline$\delta$ (B1950) of optical center & $+25^{\circ} 57^{\prime} 30^{\prime \prime}$ & Dressel \& Condon (1976) \\
\hline$D_{25}$ & $3.0 \pm 0.2$ & de Vaucouleurs et al. (1991) \\
\hline Adopted distance & $20 \mathrm{Mpc}^{\mathrm{a}}$ & Schulman et al. (1994) \\
\hline$B$ & $13.64 \pm 0.02$ & Schulman (1995) \\
\hline$R$ & $12.51 \pm 0.02$ & Schulman (1995) \\
\hline$L_{B}$ & $3.3 \pm 0.7 \times 10^{8} L_{\odot}^{\mathrm{b}}$ & Schulman (1995) \\
\hline$L_{\mathrm{H} \alpha}$ & $1.5 \pm 0.5 \times 10^{7} L_{\odot}^{\mathrm{b}}$ & Schulman (1995) \\
\hline$L_{\mathrm{FIR}}$ & $7.8 \pm 3.6 \times 10^{7} L_{\odot}$ & Schulman (1995) \\
\hline Heliocentric systemic velocity & $749 \pm 2 \mathrm{~km} \mathrm{~s}^{-1}$ & This paper \\
\hline Rotational velocity, $v_{\text {rot }}$ & $90 \mathrm{~km} \mathrm{~s}^{-1}$ & This paper \\
\hline Inclination of inner disk & $33^{\circ}$ & This paper \\
\hline PA of inner disk major axis & $10-15^{\circ}$ & This paper \\
\hline Maximum $\mathrm{H}$ I radius at $N_{\mathrm{HI}}=10^{19} \mathrm{~cm}^{-2}$ & $5.8=35 \mathrm{kpc}$ & This paper \\
\hline Total H I linewidth (FWHM) & $120 \mathrm{~km} \mathrm{~s}^{-1}$ & This paper \\
\hline Total $\mathrm{H}$ I integrated flux density & $82 \mathrm{Jy} \mathrm{km} \mathrm{s}^{-1}$ & This paper \\
\hline Total H I mass & $7.2 \times 10^{9} M_{\odot}$ & This paper \\
\hline Arecibo H I mass & $2.0 \times 10^{9} M_{\odot}$ & Schulman et al. (1994) \\
\hline "'Corrected" Arecibo H I mass & $2.3 \times 10^{9} M_{\odot}$ & Corrected as in Hewitt et al. (1983) \\
\hline Total mass within the optical disk, $M_{\text {Tot }}$ & $1.6 \times 10^{10} M_{\odot}$ & $0.5 D_{25} \times v_{\mathrm{rot}}^{2} / G$ \\
\hline$M_{\mathrm{Tot}} / L_{B}$ & $7.3 M_{\odot} / L_{\odot, B}^{c}$ & This paper \\
\hline Arecibo H I mass to $M_{\mathrm{Tot}}$ ratio, " $M_{\mathrm{H} \mathrm{I}} / M_{\mathrm{Tot}}$ ", & 0.14 & Calculated as in Roberts \& Haynes (1994) \\
\hline
\end{tabular}

${ }^{a}$ Consistent with $H_{0}=50 \mathrm{~km} \mathrm{~s}^{-1} \mathrm{Mpc}^{-1}$.

${ }^{\mathrm{b}}$ Corrected for Galactic and internal extinction.

${ }^{c}$ Uncorrected for Galactic or internal extinction. $L_{\odot, B}=5 \times 10^{32}$ ergs s$^{-1}$.

file; these wings are the expected signature of HVCs in a disk galaxy (Schulman et al. 1994). The four galaxies without high-velocity wings have low star formation rates, as determined from optical and far-infrared observations (Schulman et al. 1994; Schulman 1995). These filledaperture observations are very suggestive, but they require confirmation and do not in any case contain information on the spatial distribution of the high-velocity gas.

Very Large Array (VLA) ${ }^{4}$ observations of NGC 5668, one of the galaxies with high-velocity wings, revealed more than $4 \times 10^{8} M_{\odot}$ of high-velocity neutral hydrogen, most of which is located primarily outside the optical disk of the galaxy and may be infalling material comparable to the Magellanic Stream in our own Galaxy (Schulman et al. 1996). About $40 \%$ of the high-velocity gas that was detected with Arecibo is located within the optical disk and may be halo gas produced by a galactic fountain. The VLA observations of NGC 5668 also reveal an increased H I velocity dispersion within the optical radius of the galaxy. Increased velocity dispersions have also been found within the optical disks of NGC 1058 (Dickey et al. 1990) and NGC 6946 (Kamphuis \& Sancisi 1993; Boulanger \& Viallefond 1992). Massive stars and young regions of star formation transmit energy to the gas via expanding $\mathrm{H}$ II regions, stellar winds, and supernova explosions (Elmegreen \& Thomasson 1993), which could result in larger $\mathrm{H}$ I velocity dispersions within the optical disks of galaxies with active star formation. The global velocity dispersion of cold gas in models of the Galactic ISM

${ }^{4}$ The VLA is operated by the National Radio Astronomy Observatory, which is a facility of the National Science Foundation operated under cooperative agreement by Associated Universities, Inc. increases as a function of energy injection rate from stellar winds and/or supernovae (Rosen \& Bregman 1995).

High-velocity material could lie "between the horns" of the filled-aperture $\mathrm{H}$ I profiles of galaxies, resulting in singledish spectra without high-velocity wings for galaxies that do have high-velocity gas. If this occurs, the conclusion that galaxies without active star formation lack high-velocity clouds (Schulman et al. 1994) would be premature. This is important because the presence of active star formation is the only galaxian property that has compelling evidence for a link to the presence of high-velocity neutral hydrogen in the Arecibo observations. Because the distribution and kinematics of neutral hydrogen in galaxies are inherently complex, it is important to be sure that any $\mathrm{H}$ I that appears to be HVCs in external galaxies is not due to warped and flared disks or to inherent asymmetries in the distribution of neutral hydrogen. It is therefore necessary to test the process of modeling spiral galaxies that is used to determine the amount of HVCs in galaxies that have high-velocity wings. These issues can be addressed through VLA observations of a galaxy in the Arecibo sample that does not have high-velocity wings.

This paper reports on sensitive $21 \mathrm{~cm}$ observations of UGC 12732 obtained with the VLA for comparison with earlier observations of NGC 5668. Table 1 lists some general information about UGC 12732. The high-sensitivity Arecibo spectrum of UGC 12732 shows no detected high-velocity wings (see Figs. 11(a) and 11(b)), suggesting that this galaxy does not have a disk-wide population of high-velocity clouds (HVCs). The star formation rate in UGC 12732 is an order of magnitude lower than that of NGC 5668, although the galaxies have similar neutral hydrogen masses and sizes. UGC 
TABLE 2. Observational setup.

\begin{tabular}{|c|c|c|c|c|}
\hline Parameter & \multicolumn{4}{|c|}{ Value } \\
\hline Object & \multicolumn{4}{|c|}{ UGC 12732} \\
\hline Instrument & \multicolumn{4}{|c|}{ Very Large Array (VLA) } \\
\hline Configuration & \multicolumn{4}{|c|}{ D Array } \\
\hline Total observing time & \multicolumn{4}{|c|}{$3 \times 7.5$ hours $+1 \times 2.5$ hours } \\
\hline Observation dates (1992) & 14 Aug & 15 Aug & 17 Aug & 23 Sep \\
\hline Time on source (hours) & 5.7 & 2.9 & 4.4 & $1.7^{2}$ \\
\hline \multirow[t]{2}{*}{ Average number of antennas } & 26 & 21 & 20 & 26 \\
\hline & \multicolumn{2}{|c|}{$\alpha(\mathrm{B} 1950.0)$} & \multicolumn{2}{|c|}{$\delta(\mathrm{B} 1950.0)$} \\
\hline Field center & \multicolumn{2}{|c|}{$23^{\mathrm{h}} 38^{\mathrm{m}} 00^{\mathrm{s}}$} & \multicolumn{2}{|c|}{$+25^{\circ} 57^{\prime} 00^{\prime \prime}$} \\
\hline Flux density calibrator & \multicolumn{4}{|c|}{$3 \mathrm{C} 48$} \\
\hline Phase calibrator & \multicolumn{4}{|c|}{$\mathrm{PKS} \mathrm{B} 2251+244$} \\
\hline Central velocity of spectral band & \multicolumn{4}{|c|}{$750 \mathrm{~km} \mathrm{~s}^{-1}$} \\
\hline Observed baselines (min-max) & \multicolumn{4}{|c|}{$35 m-1030 m$} \\
\hline FWHP of primary beam & \multicolumn{4}{|c|}{$32^{\prime}$} \\
\hline System temperature & \multicolumn{4}{|c|}{$60 \mathrm{~K}$} \\
\hline Total bandwidth & \multicolumn{2}{|c|}{$3.125 \mathrm{MHz}$} & \multicolumn{2}{|c|}{$652 \mathrm{~km} \mathrm{~s}^{-1}$} \\
\hline Number of channels & \multicolumn{4}{|c|}{64 (Hanning smoothing applied) } \\
\hline Channel spacing & \multicolumn{4}{|c|}{$10.3 \mathrm{~km} \mathrm{~s}^{-1}$} \\
\hline FWHM velocity resolution & \multicolumn{4}{|c|}{$10.3 \mathrm{~km} \mathrm{~s}^{-1}$} \\
\hline Type of weighting applied to $u v-$ data & \multicolumn{2}{|c|}{ natural } & \multicolumn{2}{|c|}{ uniform } \\
\hline FWHP of synthesized beam & \multicolumn{2}{|c|}{$55^{\prime \prime} \times 54^{\prime \prime}$} & \multicolumn{2}{|c|}{$31^{\prime \prime} \times 31^{\prime \prime}$} \\
\hline & \multicolumn{2}{|c|}{$5.3 \mathrm{kpc} \times 5.2 \mathrm{kpc}$} & \multicolumn{2}{|c|}{$3.0 \mathrm{kpc} \times 3.0 \mathrm{kpc}$} \\
\hline RMS noise per channel & \multicolumn{2}{|c|}{$0.30 \mathrm{mJy}$ beam $^{-1}$} & \multicolumn{2}{|c|}{$0.45 \mathrm{mJy}^{\text {beam }^{-1}}$} \\
\hline Conversion factor $\mathrm{T}_{B}(\mathrm{~K}) / \mathrm{S}(\mathrm{mJy})$ & \multicolumn{2}{|c|}{0.20} & \multicolumn{2}{|c|}{0.63} \\
\hline RMS column density of integrated HI map & \multicolumn{2}{|c|}{$3.4 \times 10^{18} \mathrm{~cm}^{-2}$} & \multicolumn{2}{|c|}{$1.6 \times 10^{19} \mathrm{~cm}^{-2}$} \\
\hline
\end{tabular}

12732 does have a smaller optical size than NGC 5668 and a larger $\mathrm{HI}$ size to optical size ratio than most galaxies $\left(R_{\mathrm{H}, \max } / R_{25}=3.5\right.$, while Salpeter \& Hoffman 1996 found a mean of $R_{\mathrm{H} \text {,max }} / R_{25}=2.7$ for $107 \mathrm{Sdm}, \mathrm{Sm}, \mathrm{Im}$, and BCD galaxies). This may indicate that star formation has been less efficient in UGC 12732 than in most late-type spirals. UGC 12732 does not have a larger H I mass fraction within the Arecibo beam when compared to galaxies of similar type; the correction from Arecibo H I flux density to "true" H I flux density used by Hewitt et al. (1983) gives $M_{\mathrm{HI}} / M_{\mathrm{T}}=$ 0.14 for UGC 12732, while Roberts \& Haynes (1994) find a median of $M_{\mathrm{HI}} / M_{\mathrm{T}}=0.15$ for $490 \mathrm{Sm}$ and Im galaxies from the UGC catalog (Nilson 1973). The "true"' H I flux density is less than the VLA H I flux density because Hewitt et al. (1983) assumed a constant $\mathrm{H}$ I-to-optical size; the scatter in this parameter is very large, and about $10 \%$ of the galaxies they mapped had H I-to-optical sizes larger than that of UGC 12732 (see their Fig. 8).

The VLA observations of UGC 12732 are presented in Sec. 2, a determination of the $\mathrm{H} \mathrm{I}$ distribution and kinematics is made in Sec. 3, and a discussion of the results and conclusions can be found in Sec. 4. The distance to UGC 12732 is assumed to be $20 \mathrm{Mpc}$, consistent with $H_{0}=50 \mathrm{~km} \mathrm{~s}^{-1}$ $\mathrm{Mpc}^{-1}$ as used in our previous papers.

\section{21 CM OBSERVATIONS}

\subsection{Data Acquisition}

The $21 \mathrm{~cm}$ observations presented here were obtained with the VLA in 1992 August and September; the observa- tional configuration is listed in Table 2 . The bandwidth used was $3.125 \mathrm{MHz}$, centered on a heliocentric velocity of 750 $\mathrm{km} \mathrm{s}^{-1}$ and divided into 64 channels, each covering $10.3 \mathrm{~km}$ $\mathrm{s}^{-1}$. The data were taken in $3 \times 7.5$ hours plus an additional 2.5 hours that were allocated to make up for data lost during the first three nights of observing. Of these 25 hours, 19 were spent observing UGC 12732. The flux/bandpass calibrator, 3C 48 (flux density $=16.08 \mathrm{Jy}$ at $1417 \mathrm{MHz}$ ), was observed for about 8 minutes at the beginning and end of each observation. The phase calibrator, PKS B2251+244 (flux density $=1.9 \mathrm{Jy}$ at $1417 \mathrm{MHz}$ ), was observed for 3 to 4 minutes every 45 minutes. Due to the many problems that occurred during the data acquisition, only 15 hours of observations of UGC 12732 contained usable data. There were an average of 23 antennas that could be used during this time. A more detailed discussion of the data acquisition problems can be found in Schulman (1995).

\subsection{Data Reduction}

Data calibration and reduction was performed using the NRAO Astronomical Imaging Processing System (AIPS), following standard procedures. Both polarizations were examined and edited for bad data and amplitude and phase calibrations were done, after which the data were bandpass calibrated. In the following section, the AIPS tasks that were used will be listed in parenthesis.

Two kinds of weighting were used in the data reduction. To optimize the sensitivity to weak, extended $\mathrm{H}$ I emission, the Fourier transformation was carried out using a natural 

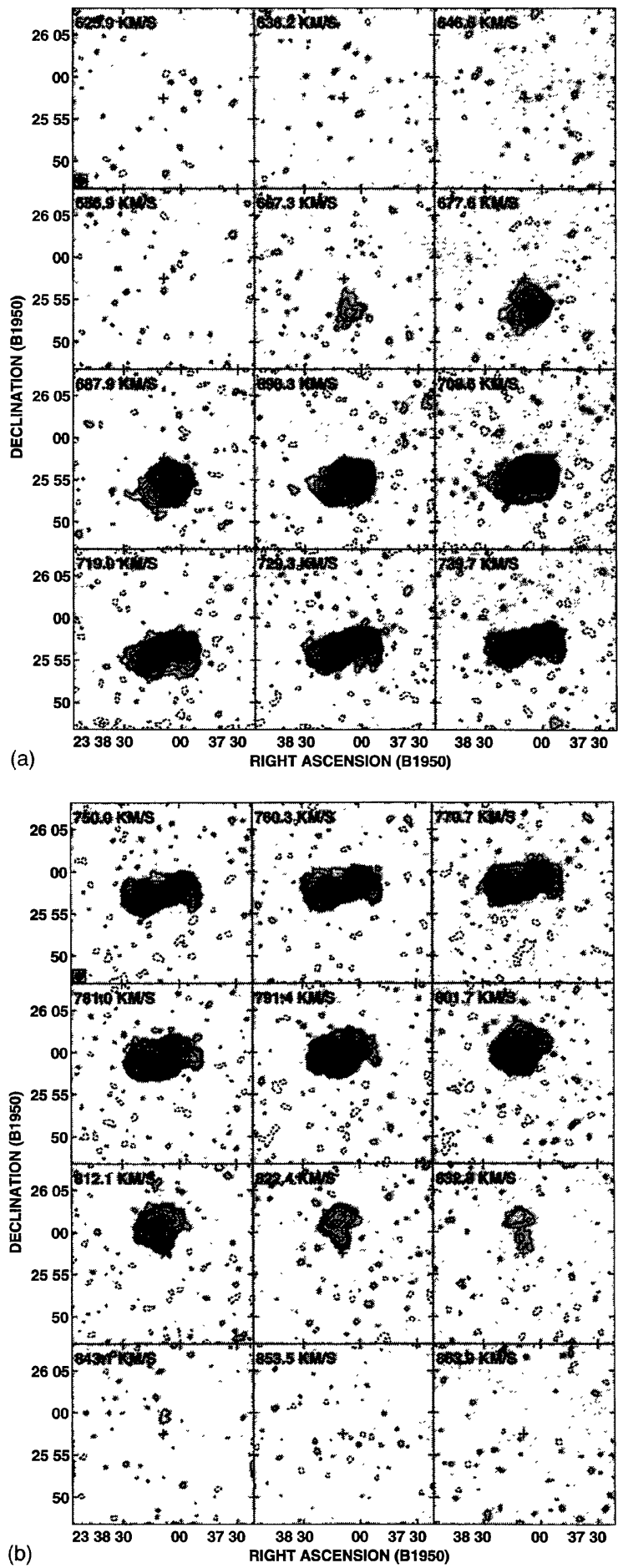

FIG. 1. Naturally-weighted H I channel maps of UGC 12732. The heliocentric radial velocities are indicated in the upper left hand corner of each map, the optical center of the galaxy is indicated with a cross and the FWHP beam is plotted in the lower left hand corner of the first channel. The contours are at $-1.5,-0.75,0.75,1.5,3,6,12,24$, and $48 \mathrm{mJy}_{\text {beam }}^{-1}$ (negative contours are dashed)

weighting for the data such that each visibility cell in the $u v$-plane was weighted according to the integration time. The short spacings are emphasized more than the larger baselines since the $u v$-tracks are more densely sampled near the center

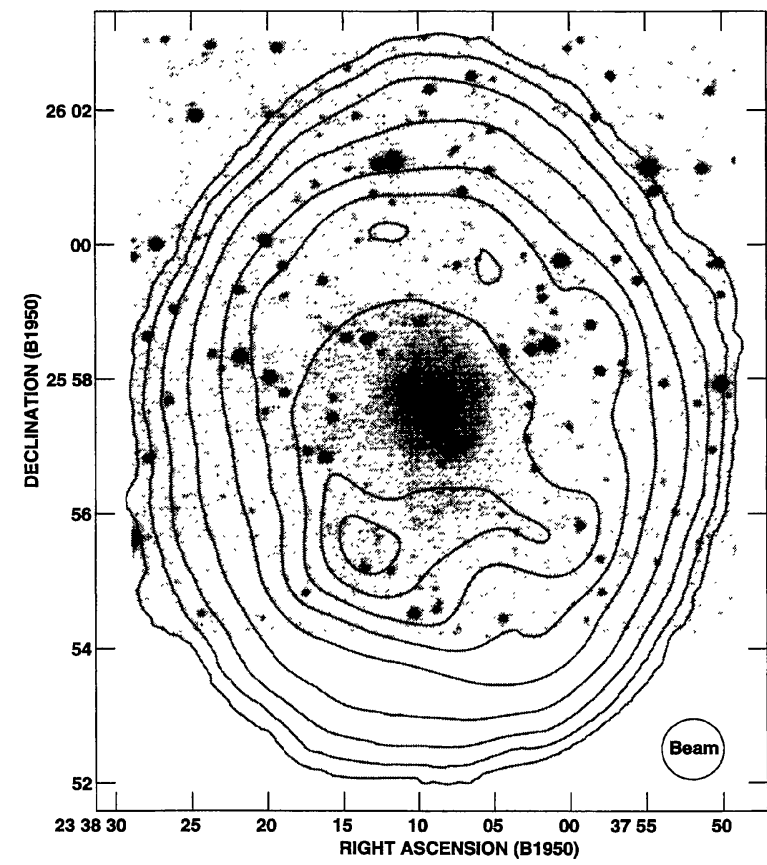

FIG. 2. Naturally-weighted total intensity H I contours of UGC 12732 (the contours are at $N_{\mathrm{H}_{\mathrm{I}}}=2,4,8,16,32,48,64,80$, and $96 \times 10^{19}$ atoms $\mathrm{cm}^{-2}$ ), superposed over a broad-band $R$ image obtained at MDM Observatory. Note that the $\mathrm{H} \mathrm{I}$ extends well beyond the optical disk of the galaxy. The $3 \sigma$ rms surface brightness limit is about $25 \mathrm{mag} \mathrm{arcsec}^{-2}$, the optical diameter at $25 \mathrm{mag} \operatorname{arcsec}^{-2}$ is about $3^{\prime}$, and the $\mathrm{H}$ I diameter at $10^{19}$ atoms $\mathrm{cm}^{-2}$ is 11:6. The VLA FWHP beam is plotted in the lower right corner.

of the $u v$-plane. In order to make maps that emphasize emission structures on a smaller scale, the Fourier transformation was carried out using a uniform weighting for the data such that each visibility cell in the $u v$-plane received equal weight, regardless of the integration time invested in it. Since the primary purpose of this project is the search for weak highvelocity features, the naturally-weighted maps will be emphasized here, but the uniformly-weighted maps are useful in showing that the neutral hydrogen is not smoothly distributed at large column densities in UGC 12732.

Before combining the four nights of observations, a strong $(\sim 7 \mathrm{Jy})$ continuum source located about a degree to the northwest of UGC 12732 had to be subtracted from the data. This source, 3C 465, is associated with NGC 7720, the $\mathrm{cD}$ galaxy in Abell $2634\left(v=9150 \mathrm{~km} \mathrm{~s}^{-1}\right.$; Pinkney et al. 1993). The area around the source in the August 14 observation was CLEANed (Högbom 1974; Clark 1980) and the resulting clean components were subtracted in the $u v$-plane from each of the four nights of data (UVSUB). The four nights of data were combined in the $u v$-plane (DBCON) and preliminary data cubes of both weightings were made (HORUS). The first three and last seven channels in frequency were discarded because the low gain at frequencies near the edge of the bandpass produces a large rms noise. The channels with $502 \leqslant v \leqslant 626 \mathrm{~km} \mathrm{~s}^{-1}$ and $864 \leqslant v \leqslant 1040 \mathrm{~km} \mathrm{~s}^{-1}$ were determined to be line-free, and these channels determined the continuum that was subtracted in the $u v$-plane (UVLIN; Cornwell et al. 1992). Data cubes were made and CLEANed 


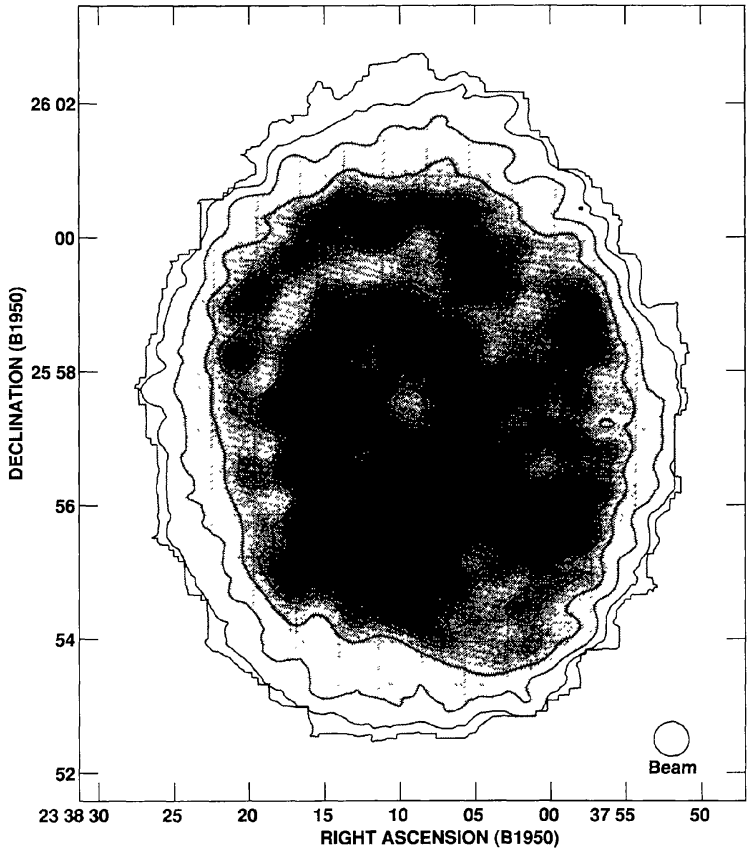

FIG. 3. Uniformly-weighted total intensity $\mathrm{HI}$ greyscale and contours of UGC 12732 (the contours are at $N_{\mathrm{H}_{1}}=4,8,16,32,48,64,80,96$, and $112 \times 10^{19}$ atoms $\mathrm{cm}^{-2}$ ). The FWHP beam is plotted in the lower right corner.

(MX). The uniformly-weighted CLEANed channel maps have a nearly uniform sensitivity $\sigma \sim 0.45 \mathrm{mJy}$ beam $^{-1}$ with a beam size (FWHP) of $31^{\prime \prime}$, while the naturally-weighted CLEANed channel maps have a nearly uniform sensitivity $\sigma \sim 0.3 \mathrm{mJy}^{-}$beam $^{-1}$ with a beam size (FWHP) of $54^{\prime \prime}$; the sensitivity of both cubes is comparable to the theoretical values. The naturally-weighted channel maps are shown in Fig. 1.

In order to create useful moment maps, it is necessary to eliminate as much noise as possible from the image cubes. The cubes were blanked at the $+2.5 \sigma$ level (i.e., all pixels with flux densities less than $0.75 \mathrm{mJy}^{\text {beam }^{-1}}$ in the naturally-weighted cube were excluded), after which all features that did not occur in at least two adjacent velocity channels were ignored. Moment maps were then created (XMOM). The integrated $\mathrm{HI}$ distribution (0th moment; Fig. 2) shows that the neutral hydrogen is much more extended than the optical $^{5}$ emission in UGC 12732 . The $3 \sigma$ rms surface brightness limit is about $25 \mathrm{mag} \operatorname{arcsec}^{-2}$, the optical diameter at $25 \mathrm{mag} \mathrm{arcsec}^{-2}$ is about $3^{\prime}$, and the $\mathrm{H} \mathrm{I}$ diameter at $10^{19}$ atoms $\mathrm{cm}^{-2}$ is 11.6. The galaxy is relatively regular at low neutral hydrogen column densities, but has structure at larger column densities that is most easily visible in the uniformlyweighted map (Fig. 3). This structure includes possible spiral arms (although the optical emission from these arms is very

${ }^{5}$ The $R$-band observations shown here were obtained with the MDM $1.3 \mathrm{~m}$ telescope on 1992 October 25 under non-photometric conditions. Photometric images of UGC 12732 obtained in 1993 (Schulman 1995) do not go as deep as these non-photometric observations, and so are not presented here. MDM Observatory is operated by the University of Michigan, Dartmouth College, and the Massachusetts Institute of Technology.

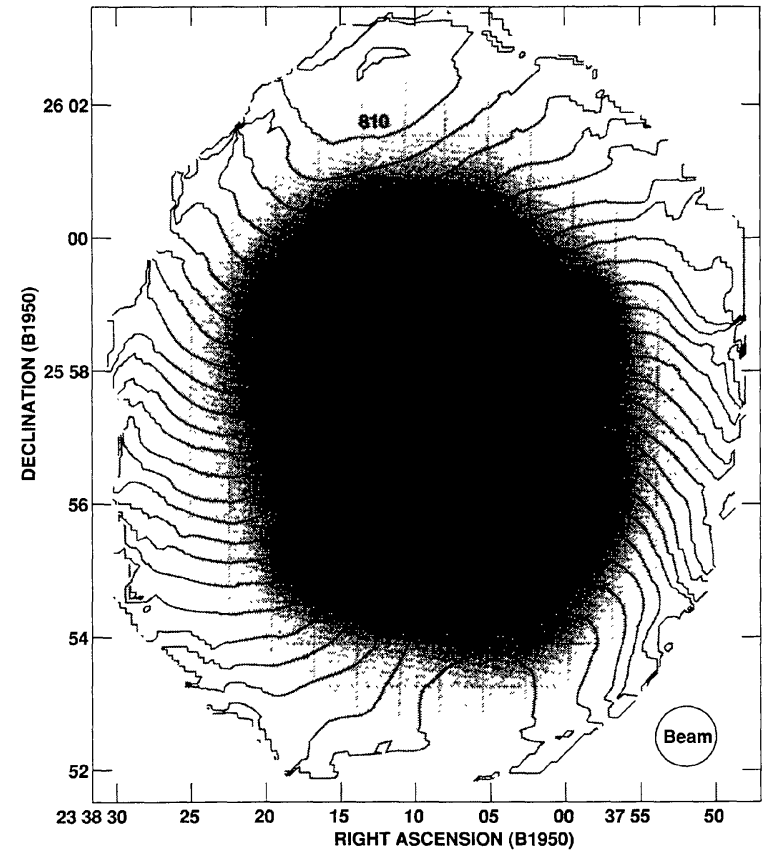

FIG. 4. The intensity-weighted mean velocity field of UGC 12732 (contours; natural weighting) superposed over the naturally-weighted total intensity $\mathrm{H} \mathrm{I}$ distribution (greyscale). There are contours every $5 \mathrm{~km} \mathrm{~s}^{-1}$ in heliocentric radial velocity from $690 \mathrm{~km} \mathrm{~s}^{-1}$ to $815 \mathrm{~km} \mathrm{~s}^{-1}$, the systemic velocity is 749 $\mathrm{km} \mathrm{s}^{-1}$, and larger velocities are to the north (the contours at $695 \mathrm{~km} \mathrm{~s}^{-1}$ and $810 \mathrm{~km} \mathrm{~s}^{-1}$ are indicated on the map). The FWHP beam is plotted in the lower right corner, and the FWHM velocity resolution is $10.3 \mathrm{~km} \mathrm{~s}^{-1}$.

faint) and a central hole in the $\mathrm{H} \mathrm{I}$ distribution. The total $\mathrm{H} \mathrm{I}$ mass is $7.2 \times 10^{9} M_{\odot}$; the $\mathrm{H}$ I mass within the Arecibo beam (see Sec. 4) is $2.2 \times 10^{9} M_{\odot}$, very similar to the mass of $2.0 \times 10^{9} M_{\odot}$ determined from the Arecibo observations of Schulman et al. (1994). The velocity field (1st moment; Fig. 4) has slightly twisted isovelocity contours outside of the optical radius, indicating that the galaxy has a kinematic warp.

The naturally-weighted, CLEANed cube was rotated by $15^{\circ}$ to align the major axis with the pixel grid, after which the cube was transposed so as to obtain a cube in (position along major axis)-(velocity)-(position along minor axis). The position-velocity diagram along the major axis (Fig. 5) shows that the $\mathrm{HI}$ velocity dispersion is roughly constant with radius, and that there is little or no high-velocity neutral hydrogen in the galaxy. Such material would be farther than $40-50 \mathrm{~km} \mathrm{~s}^{-1}$ away from the local rotational velocity.

\section{NEUTRAL HYDROGEN KINEMATICS AND DYNAMICS}

\subsection{Fitting the Velocity Field}

Tilted rings fitted to the velocity field using the task ROTCUR (Begeman 1989) in the Groningen Image Processing System (GIPSY) were used to study the kinematics of the neutral hydrogen in UGC 12732 . This task derives the rotational velocity $\left(v_{\text {rot }}\right)$, inclination $(i)$, and position angle (PA) of the galaxy as a function of radius. After the systemic velocity and central position of the galaxy were determined, 


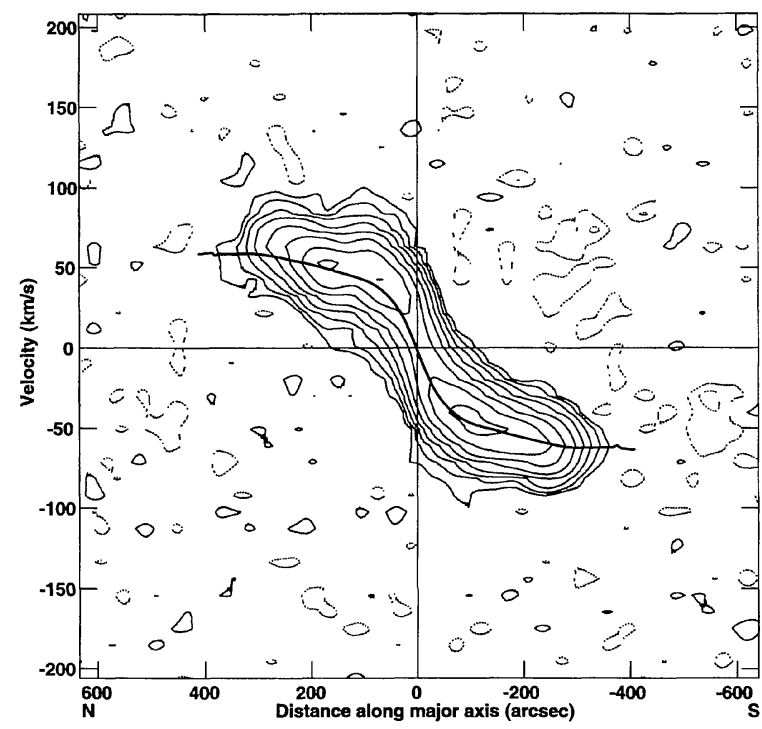

FIG. 5. A position-velocity diagram taken along the major axis of UGC 12732 through the naturally weighted data cube. The rotation curve is indicated by the solid line, and the contours are at $-1,-0.5,0.5,1,2,4,8,16$, 32 , and $64 \mathrm{mJy}^{\text {beam }}{ }^{-1}$ (negative contours are dashed).

$v_{\text {rot }}, i$, and PA were derived in an iterative fashion for the entire galaxy, keeping all but one parameter fixed for each run. The final values are shown in Fig. 6 and in Table 3. The approaching and receding sides of the galaxy were also fit separately with ROTCUR (the inputs were the solid lines in Fig. 6). Such fits allow an estimate of the errors in the solution to be made, although true asymmetries in the neutral hydrogen distribution appear as well. For clarity of presentation in Figs. 6 and 7, the radii of the rings to be fitted were shifted by $5^{\prime \prime}$ (positive for the receding, negative for the approaching side of the galaxy). Although the convention is to fit both sides of a galaxy at the same radius and then to shift the plotted points, the results of this method were misleading. Note that due to beam smearing the observed values of $v_{\text {rot }}, i$, and PA need to be corrected in order to obtain the intrinsic values (the dotted lines in Fig. 6 denote $v_{\text {rot }}$ and $i$ after correcting for beam smearing, as do the "model galaxy inputs" in Table 3; see below).
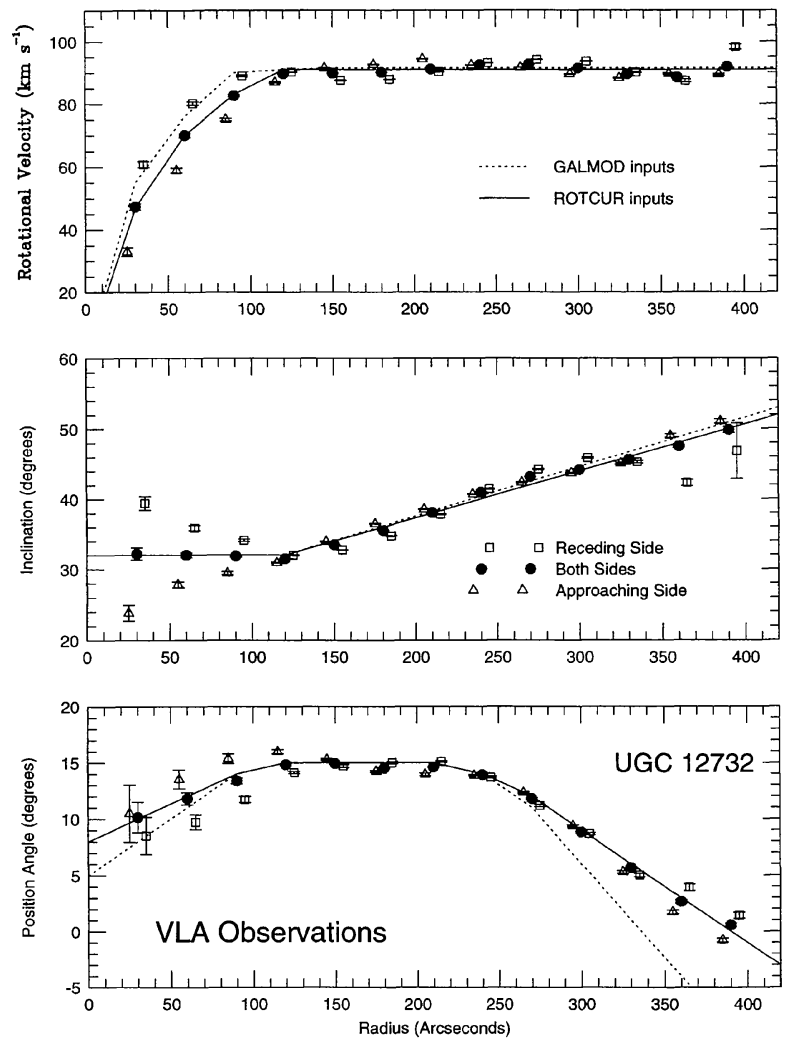

FIG. 6. Tilted-ring (ROTCUR) fits to the velocity field of UGC 12732, showing $v_{\text {rot }}, i$, and PA for each $30^{\prime \prime}$ ring. The solid lines show the inputs to ROTCUR, the dotted lines show $v_{\text {rot }}, i$, and PA after correcting for beam smearing (these are the inputs to GALMOD); the symbols are the output from ROTCUR fits to the galaxy (filled circles are from fits to the entire velocity field, open triangles are from fits to the approaching side of the galaxy, and open squares are from fits to the receding side of the galaxy). See text for details.

\subsection{Modeling UGC 12732}

In order to search for high-velocity material at low column densities, a model galaxy was created that could be compared to the observations of UGC 12732. This model galaxy can account for apparent high-velocity gas due to a warped $\mathrm{H}$ I disk, and can also include gas with different ve-

TABLE 3. Results of tilted ring and model galaxy analysis.

\begin{tabular}{|c|c|c|c|c|c|c|}
\hline \multirow[b]{2}{*}{ Radius } & \multirow[b]{2}{*}{$v_{\text {rot }}$} & \multicolumn{2}{|c|}{ Tilted ring output } & \multicolumn{3}{|c|}{ Model galaxy inputs ${ }^{\mathrm{a}}$} \\
\hline & & $i$ & PA & $v_{\text {rot }}$ & $i$ & $\mathrm{PA}$ \\
\hline $30^{\prime \prime}$ & $45.4 \pm 0.7 \mathrm{~km} \mathrm{~s}^{-1}$ & $30: 6 \pm 0: 6$ & $10^{\circ} 9 \pm 1: 0$ & $55 \mathrm{~km} \mathrm{~s}^{-1}$ & $32^{\circ}$ & $8^{\circ}$ \\
\hline 60 & $70.0 \pm 0.3$ & $32.0 \pm 0.2$ & $12.2 \pm 0.3$ & 76 & 32 & 11 \\
\hline 90 & $83.3 \pm 0.2$ & $32.1 \pm 0.1$ & $13.7 \pm 0.1$ & 90 & 32 & 14 \\
\hline 120 & $90.0 \pm 0.1$ & $31.6 \pm 0.1$ & $14.7 \pm 0.1$ & 91 & 32 & 15 \\
\hline 150 & $90.4 \pm 0.1$ & $33.7 \pm 0.1$ & $14.9 \pm 0.1$ & 92 & 34 & 15 \\
\hline 180 & $90.8 \pm 0.1$ & $35.9 \pm 0.1$ & $14.8 \pm 0.1$ & 92 & 36 & 15 \\
\hline 210 & $90.7 \pm 0.1$ & $37.9 \pm 0.1$ & $14.4 \pm 0.1$ & 92 & 38 & 15 \\
\hline 240 & $90.7 \pm 0.1$ & $39.9 \pm 0.1$ & $13.3 \pm 0.1$ & 92 & 40 & 14 \\
\hline 270 & $90.8 \pm 0.1$ & $42.0 \pm 0.1$ & $11.4 \pm 0.1$ & 92 & 43 & 11 \\
\hline 300 & $91.0 \pm 0.1$ & $43.8 \pm 0.1$ & $8.6 \pm 0.1$ & 92 & 45 & 6 \\
\hline 330 & $91.3 \pm 0.1$ & $45.9 \pm 0.1$ & $6.0 \pm 0.1$ & 92 & 49 & 1 \\
\hline 360 & $91.9 \pm 0.1$ & $47.7 \pm 0.1$ & $3.6 \pm 0.1$ & 92 & 51 & -4 \\
\hline 390 & $93.0 \pm 0.2$ & $50.0 \pm 0.2$ & $0.0 \pm 0.1$ & 92 & 53 & -9 \\
\hline
\end{tabular}

These values have effectively been corrected for beam-smearing. 

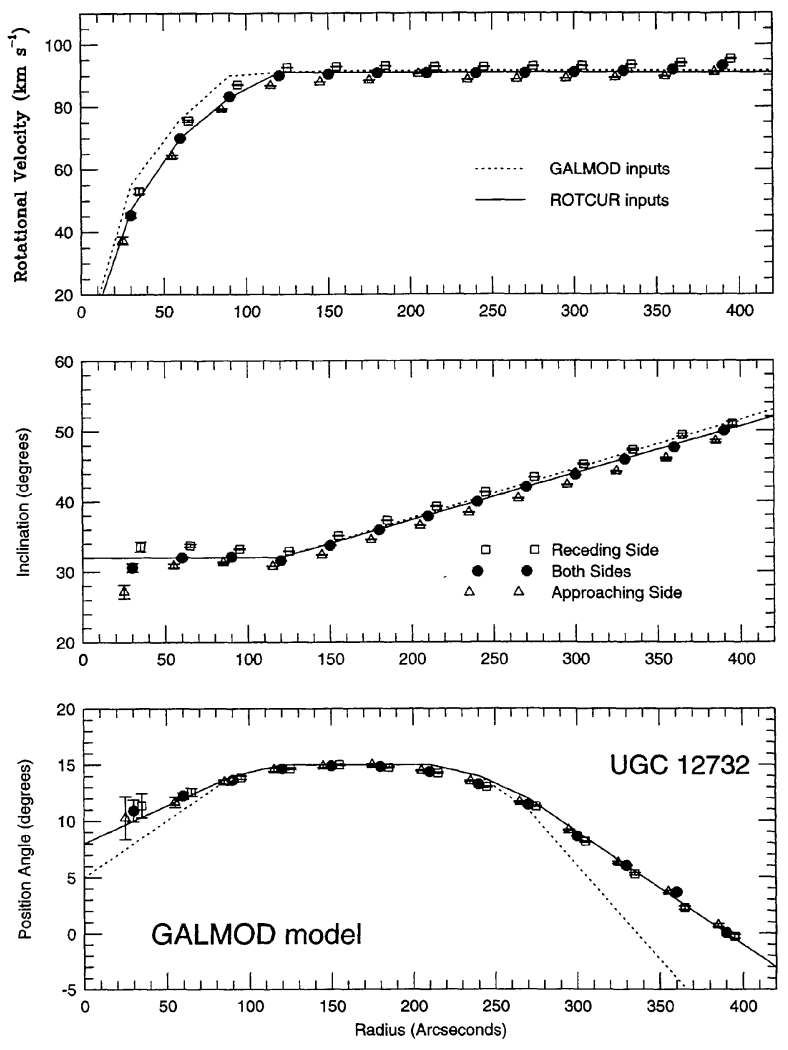

FIG. 7. Tilted-ring (ROTCUR) fits to the velocity field of the GALMOD model of UGC 12732, showing $v_{\mathrm{rot}}, i$, and PA for each $30^{\prime \prime}$ tilted ring. The solid lines show the inputs to ROTCUR, the dotted lines show the $v_{\text {rot }}, i$, and PA after correcting for beam smearing (these are the inputs to GALMOD); the symbols are the output from the ROTCUR fits to the model galaxy (filled circles are from fits to the entire velocity field, open triangles are from fits to the approaching side of the model galaxy, and open squares are from fits to the receding side of the model galaxy). See text for details.

locity dispersions at different radii. The model was constructed using the GIPSY task GALMOD; it consists of neutral hydrogen in circular orbits on rings of different radii and is convolved with the VLA synthesized beam for comparison with the VLA observations. Each ring is characterized by a velocity dispersion, a scale height perpendicular to the plane of the ring, a rotational velocity, an inclination, a position angle, and a column density. The central "ring", is actually a disk with a radius of $30^{\prime \prime}$. All rings share the same systemic velocity and central position. A constant velocity dispersion of $9 \pm 1 \mathrm{~km} \mathrm{~s}^{-1}$ fits the observations of UGC 12732; the uncertainty in the velocity dispersion comes primarily from the velocity $\left(\sigma_{v}=4.4 \mathrm{~km} \mathrm{~s}^{-1}\right)$ and spatial $\left(\theta_{\mathrm{FWHP}}=5.3 \mathrm{kpc}\right)$ resolution of the observations. The scale height for the $\mathrm{H}$ was assumed to be constant within $12.5 \mathrm{kpc}$ of the center and to increase linearly with radius at larger distances from the center, as is true for our Galaxy (Kulkarni et al. 1982) and for M31 (Brinks \& Burton 1984); the models are not very sensitive to the scale height of the gas. A disk scale length was not assumed; instead, the initial $\mathrm{H}$ I column density as a function of radius was determined by fitting ellipses to the total intensity map (Fig. 2) and correcting for the inclination of the galaxy. The input $\mathrm{H}$ I column density distribution was

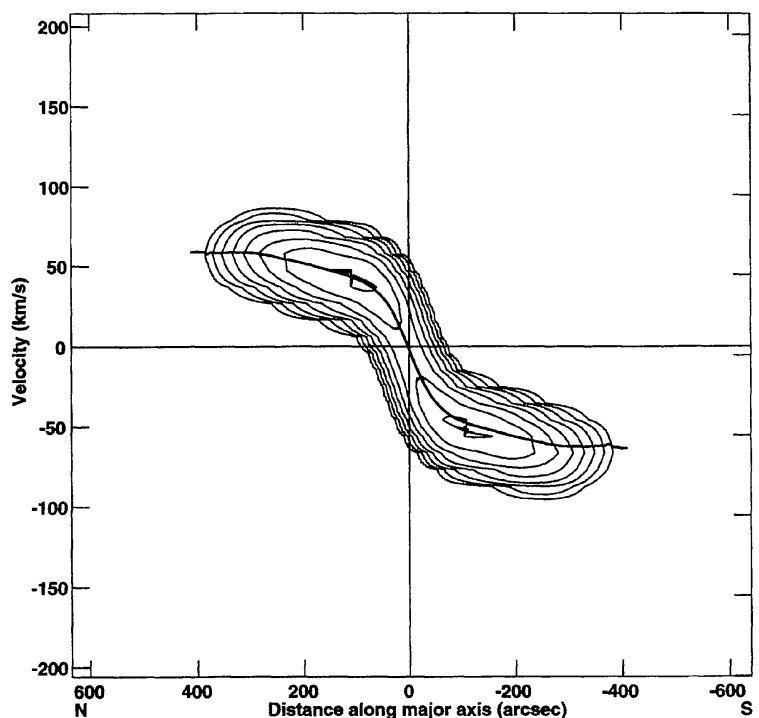

FIG. 8. A position-velocity diagram taken along the major axis of the GALMOD model of UGC 12732. The rotation curve is indicated by the solid line, and the contours are at $0.5,1,2,4,8,16,32$, and $64 \mathrm{mJy}^{\text {beam }}{ }^{-1}$. Note the similarity to the observations (Fig. 5).

adjusted until the output $\mathrm{HI}$ column density distribution matched that of the azimuthally averaged observations. Initial guesses for the rotational velocity, inclination, and position angle as a function of radius were taken from the tiltedring (ROTCUR) fits to the observed velocity field (Fig. 6). Since these parameters are beamwidth-smeared, the model galaxy inputs were adjusted until the tilted-ring (ROTCUR) fits to the model matched the fits to the observations. The major

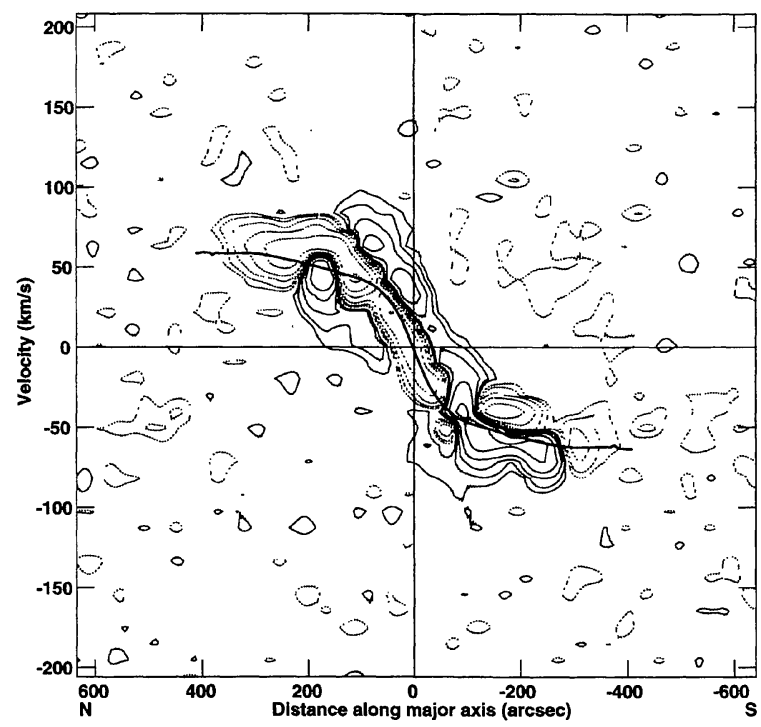

FIG. 9. Residual position-velocity diagram along the major axis of UGC 12732 , obtained by subtracting Fig. 8 from Fig. 5. The rotation curve is indicated by the solid line, and the contours are at $-16,-8,-4,-2,-1$, $-0.5,0.5,1,2,4$, and $8 \mathrm{mJy}_{\text {beam }}^{-1}$ (negative contours are dashed). Highvelocity gas would appear as material at velocities greater than 40 to $50 \mathrm{~km}$ $\mathrm{s}^{-1}$ with respect to the local rotational velocity; there is no significant highvelocity material present. 


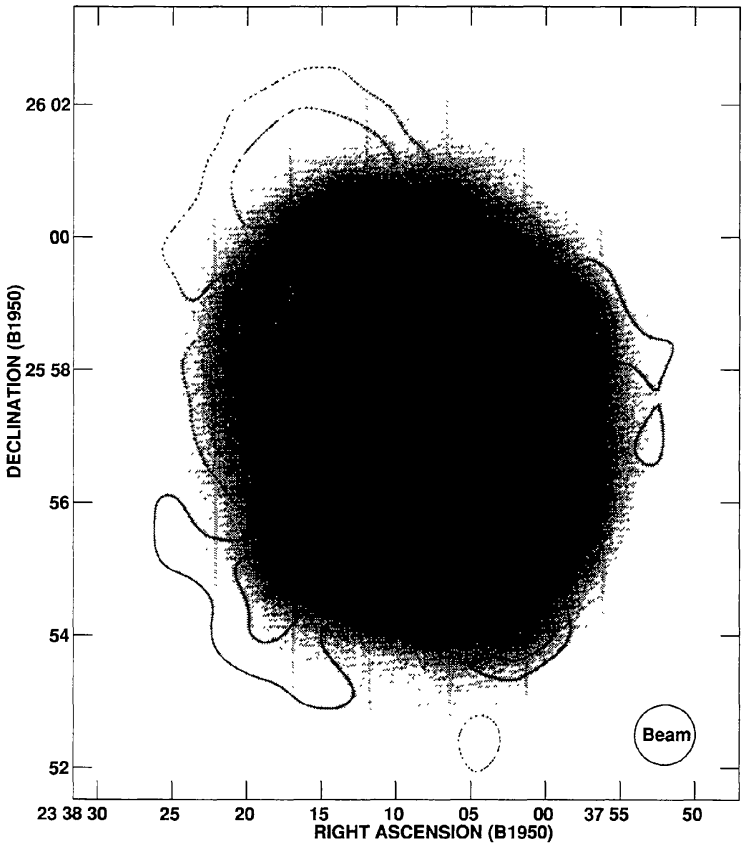

FIG. 10. Residual total intensity H I map of UGC 12732 (the contours are at $N_{\mathrm{H}}=-16,-8,-4,4,8,16$ and $32 \times 10^{19}$ atoms $\mathrm{cm}^{-2}$ with negative contours dashed), superposed over the observed total intensity $\mathrm{H}$ I map. The model is azimuthally symmetric while the real galaxy is not, leading to the residuals, which are positive if the observed integrated flux density is larger than the model integrated flux density. The H I FWHP beam is plotted in the lower right hand corner.

modifications were an increased input rotational velocity from $0^{\prime \prime}$ to $90^{\prime \prime}$ and steeper changes in position angle with radius between 0 "to $90^{\prime \prime}$ and at radii larger than $240^{\prime \prime}$. The rotational velocity, inclination, and position angle of the model are shown in Fig. 7 with the model galaxy (GALMOD) inputs shown as dotted lines; these inputs are also presented in Table 3.

The position-velocity diagram along the major axis of the GALMOD model is shown in Fig. 8, and is very similar to that of the VLA observations (Fig. 5). The difference between the galaxy and the model is shown in Fig. 9; the residuals are typically $25 \%$ of the observations. The residual total intensity contours, shown in Fig. 10 superposed over the observed total intensity image, are dominated by two features: a region in the south in which the column densities in the galaxy are larger than the column densities in the model, and a region in the north in which the column densities in the galaxy are smaller than the column densities in the model. These azimuthal asymmetries can be seen in Fig. 3. The mean of the absolute value of the ratio of the residual emission to the observed emission is $12 \% \pm 9 \%$; the maximum residual-toobserved ratio is $38 \%$. Note that the goal of this paper is not to make a model that will match the observations perfectly, but rather to use a relatively simple model to aid in the search for high-velocity neutral hydrogen. We searched for emission that is farther than $40-50 \mathrm{~km} \mathrm{~s}^{-1}$ away from the local rotational velocity in position-velocity space, and set an upper limit of $2 \times 10^{7} M \odot$ to the mass in such high-velocity neutral hydrogen. This corresponds to less than $0.3 \%$ of the total amount of neutral hydrogen in UGC 12732. The sensi-
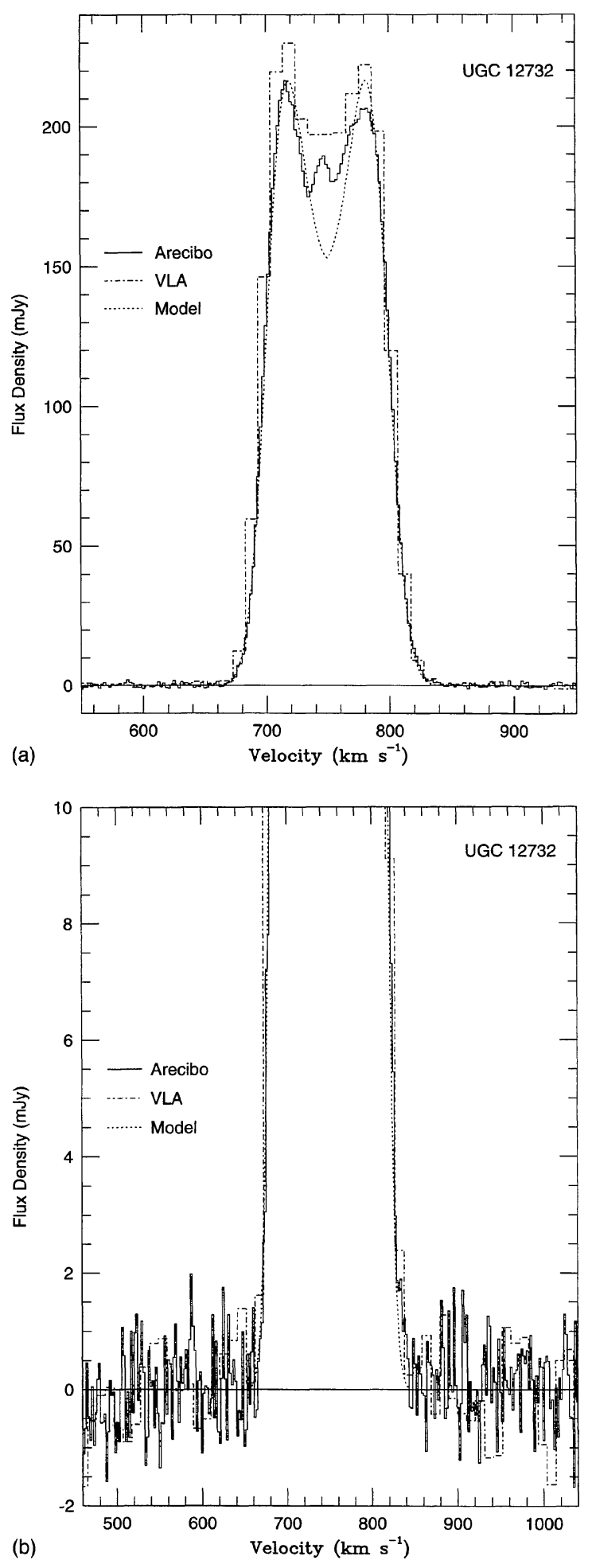

FIG. 11. The H I profile of UGC 12732. The Arecibo spectrum has $2 \mathrm{~km}$ $\mathrm{s}^{-1}$ channels (solid histogram) and the VLA spectrum (dash-dot histogram) has $10 \mathrm{~km} \mathrm{~s}^{-1}$ channels. The VLA data were convolved with the Arecibo beam for comparison with the Arecibo spectrum. A spectrum for a disk galaxy model with no high-velocity gas (Schulman et al. 1994) is also plotted (dotted line). High-velocity gas would appear as wings beyond the model (dotted line) spectrum (see Schulman et al. 1994 for details). (a) Full flux-density range. (b) Expanded y-axis. 


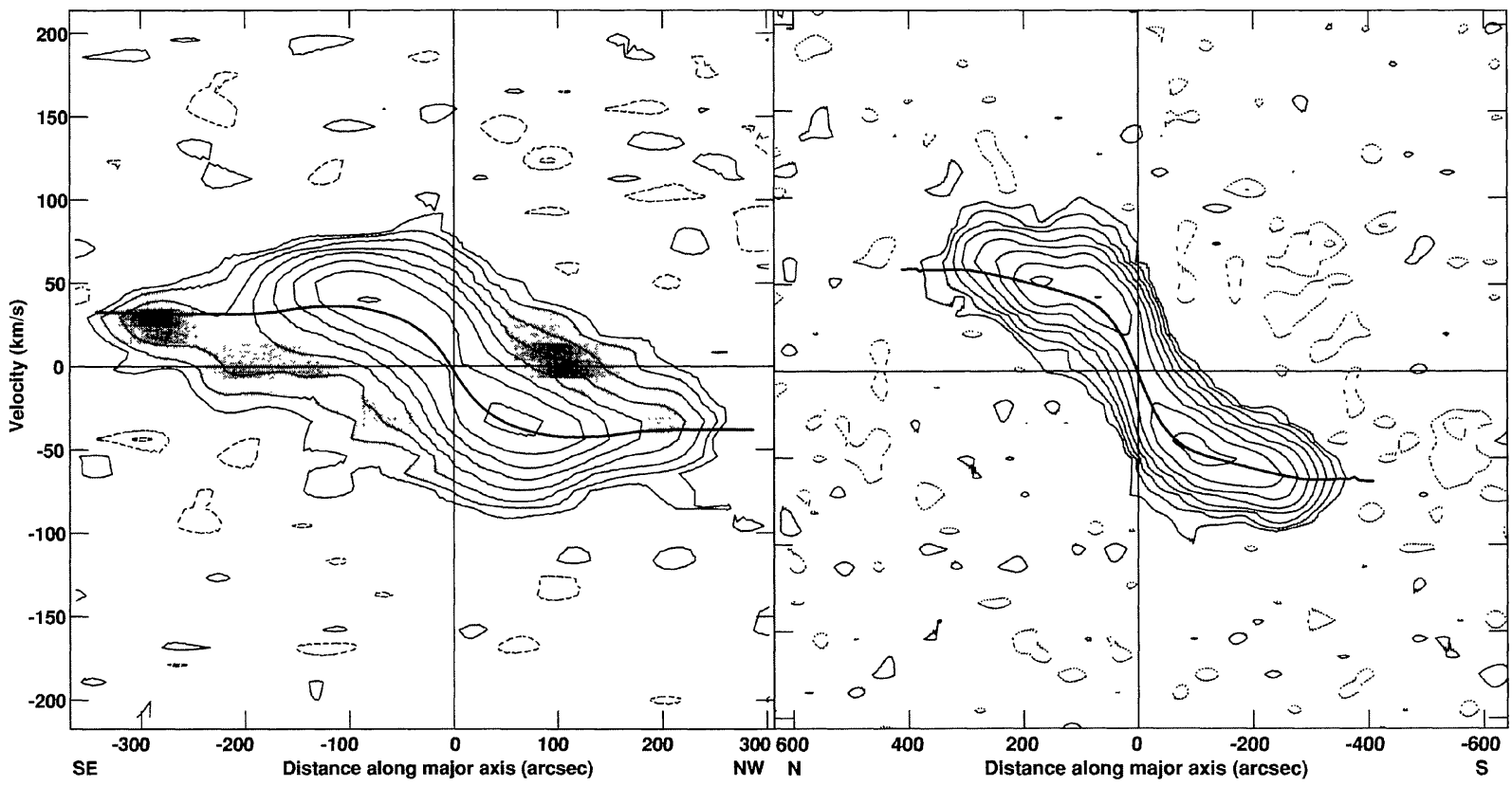

FIG. 12. Position-velocity diagrams taken along the major axes of NGC 5668 and UGC 12732 . The rotation curves are indicated by solid lines and negative contours are dashed. The contours for NGC 5668 (Fig. 4 from Schulman et al. 1996) are at $-1.3,-0.65,0.65,1.3,2.6,5.2,10.4,20.8,41.6$, and 83.2 mJy beam $^{-1}$, while the contours for UGC 12732 (Fig. 5 from this paper) are at $-1,-0.5,0.5,1,2,4,8,16,32$, and $64 \mathrm{mJy} \mathrm{beam}^{-1}$. The greyscale indicates the intensity of kinematically distinct neutral hydrogen in NGC 5668 (see Schulman et al. 1996 for details).

tivity of these observations to high-velocity gas depends on its spatial and velocity distribution; we would have detected high-velocity H I in UGC 12732 if it had surface densities of a few $\times 10^{19}$ atoms $\mathrm{cm}^{-2}$.

\section{DISCUSSION AND CONCLUSIONS}

In order to compare the VLA and Arecibo data, the VLA observations were multiplied with the beam shape of the Arecibo $21 \mathrm{~cm}$ dual circular feed. The Arecibo beam can be approximated by a Gaussian of FWHP $=3.3$ plus a $10 \%$ sidelobe ring with radii of $276^{\prime \prime}$ and $360^{\prime \prime}$ (Haynes \& Giovanelli 1984). The VLA observations recover the H I flux density and the spectral shape observed with the Arecibo telescope (Fig. 11), and so confirm the Arecibo results that there is no evidence for high-velocity wings in the spectrum of UGC 12732 (Schulman et al. 1994). Moreover, the VLA data show that there is no high-velocity $\mathrm{H}$ I hidden "between the horns," i.e., there is no $\mathrm{H}$ I which has a velocity that does not agree with that expected for its location in the disk based on galactic rotation while staying within the same velocity as the bulk of the $\mathrm{H} \mathrm{I}$ emission. This result is important because the presence of high-velocity clouds as indicated by wings to a single dish spectrum appears to be correlated with active star formation (Schulman et al. 1994; Schulman 1995). UGC 12732 has low far-infrared (Schulman et al. 1994; Schulman 1995) and $\mathrm{H} \alpha$ (Schulman 1995) luminosities, implying a low star formation rate.

The sample galaxies were selected on the basis of not having nearby companions, but it is difficult to find a truly isolated galaxy. There are galaxies with velocity differences of less than $300 \mathrm{~km} \mathrm{~s}^{-1}$ and projected distances of less than $400 \mathrm{kpc}$ from half of the galaxies in the sample. Based on our Arecibo sample, galaxies with and without high-velocity $\mathrm{H}$ I wings are equally likely to be isolated. NGC 7741, an Scd galaxy $45^{\prime}$ ( $260 \mathrm{kpc}$ for $\mathrm{D}=20 \mathrm{Mpc}$ ) away from UGC 12732 , was visible in the VLA observations, although the $\mathrm{H}$ emission was very weak because the galaxy was well outside the 32' FWHP primary beam. The systemic velocity of NGC 7741 is within $10 \mathrm{~km} \mathrm{~s}^{-1}$ of that of UGC 12732, implying that the galaxies are spatially close. Despite this possible proximity, neither galaxy appears disturbed in $\mathrm{H}$ I or optical observations. The fact that no high-velocity gas was detected suggests that these galaxies have not interacted in the recent past, and it is possible that they have not yet encountered each other.

These VLA observations and GALMOD models confirm that not all disk galaxies have high-velocity clouds of neutral hydrogen. The model of a rotating disk of neutral hydrogen with a velocity dispersion of $9 \mathrm{~km} \mathrm{~s}^{-1}$ throughout adequately reproduces the observations. The residuals left after the model is subtracted from the galaxy are substantial, but are not suggestive of an interaction or of an additional component of neutral hydrogen with a large velocity dispersion.

VLA observations of UGC 12732 and NGC 5668 confirm the Arecibo telescope observations of these two galaxies. In Schulman et al. (1996), the galaxy modeling method used here was applied to NGC 5668, which has high-velocity wings that were detected with the Arecibo telescope. A comparison of the position-velocity diagrams along the major axes of these two galaxies (Fig. 12) demonstrates the differences between them. In UGC 12732, the velocity dispersion 
is constant with radius, and there is no detected high-velocity emission. NGC 5668, on the other hand, has an abundance of high-velocity neutral hydrogen and a larger $\mathrm{H}$ I velocity dispersion within the optical radius. Although the galaxies have similar neutral hydrogen masses and sizes, the star formation rate in NGC 5668 is one to two orders of magnitude greater than the star formation rate in UGC 12732 according to $\mathrm{H} \alpha$ (Schulman 1995) and far-infrared (Schulman et al. 1994; Schulman 1995) observations. High-velocity wings were not detected in the four galaxies in the Arecibo survey that have low star formation rates, suggesting a connection between star formation and the presence of high-velocity neutral hydrogen in disk galaxies. The presence of extraplanar diffuse ionized gas (Dettmar 1993; Rand 1996) and extended radio continuum halos (Dahlem et al. 1995) are also related to the star formation rate in the disks of spiral galaxies. These results lend support to the hypothesis that at least some highvelocity clouds in disk galaxies are driven into the halo by active star formation, and that when active star formation is absent there is little mass in high-velocity clouds of neutral hydrogen.

We would like to thank Barry Clark for allocating additional VLA time to replace the observations lost due to instrumental problems. Thanks also to Kor Begeman and Flor Sicking for their assistance with GIPSY. This research has made use of the NASA/IPAC Extragalactic Database (NED), which is operated by the Jet Propulsion Laboratory, California Institute of Technology, under contract with the National Aeronautics and Space Administration. It has also made use of NASA's Astrophysics Data System Abstract Service, and of the Simbad database, operated at CDS, Strasbourg, France. This work was supported by the National Science Foundation and by the National Aeronautics and Space Administration through grants NGT-50901 (ES) and NAGW2135 (JNB).

REFERENCES

Begeman, K. 1989, A\&A, 223, 47

Boulanger, F., \& Viallefond, F. 1992, A\&A, 266, 37

Bregman, J. N. 1980, ApJ, 236, 577

Brinks, E., \& Burton, W. B. 1984, A\&A, 141, 195

Centurión, M., Vladilo, G., de Boer, K. S., Herbstmeier, U., \& Schwarz, U. J. 1994, A\&A, 292, 261

Clark, B. G. 1980, A\&A, 89, 377

Cornwell, T. J., Uson, \& Haddad, N. 1992, A\&A, 258, 583

Dahlem, M., Lisenfeld, U., \& Golla, G. 1995, ApJ, 444, 119

Danly, L., Albert, C. E., \& Kuntz, K. D. 1993, ApJ, 416, L29

Dettmar, R.-J. 1993, Rev. Mod. Astron., 6, 33

de Vaucouleurs, G., de Vaucouleurs, S., Corwin, H., Buta, R., Paturel, G., \&

Fouqué, P. 1991, Third Reference Catalogue of Bright Galaxies (Springer, New York)

Dickey, J. M., Hanson, M. M., \& Helou, G. 1990, ApJ, 352, 522

Dressel, L. L., \& Condon, J. J. 1976 ApJS, 31, 187

Elmegreen, B. G., \& Thomasson, M. 1993, A\&A, 272, 37

Haynes, M. P., \& Giovanelli, R. 1984, AJ, 89, 758

Hewitt, J. N., Haynes, M. P., \& Giovanelli, R. 1983, AJ, 88, 272

Högbom, J. A. 1974, A\&AS, 15, 417

Kamphuis, J., \& Sancisi, R. 1993, A\&A, 273, L31

Kulkarni, S. R., Blitz, L., \& Heiles, C. 1982, ApJ, 259, L63

Murai, T., \& Fujimoto, M. 1980, PASJ, 32, 581
Nilson, P. 1973, Uppsala General Catalogue of Galaxies (Royal Society of Sciences of Uppsala, Uppsala) (UGC)

Pinkney, J., Rhee, G., Burns, J. O., Hill, J. M., Oegerle, W., Batusky, D., \& Hintzen, P. 1993, ApJ, 416, 36

Rand, R. J. 1996, in The Interplay Between Massive Star Formation, the ISM, and Galaxy Evolution, edited by D. Kunth, B. Guiderdoni, M. Heydari-Malayeri, and T. X. Thuan (Editions Frontières, Gif-sur-Yvette Cedex), p. 515

Roberts, M. S., \& Haynes, M. P. 1994, ARA\&A, 32, 115

Rosen, A., \& Bregman, J. N. 1995, ApJ, 440, 634

Salpeter, E. E., \& Hoffman, G. L. 1996, ApJ, 465, 595

Schulman, E. 1995, Ph.D. thesis, University of Michigan

Schulman, E., Bregman, J. N., Brinks, E., \& Roberts, M. S. 1996, AJ, 112, 960

Schulman, E., Bregman, J. N., \& Roberts, M. S. 1994, ApJ, 423, 180

Shapiro, P. R., \& Field, G. B. 1976, ApJ, 205, 762

Wakker, B. P. 1990, Ph.D. thesis, Groningen University

Wakker, B. P. 1991, A\&A, 250, 499

Wakker, B., Howk, C., Schwarz, U., van Woerden, H., Beers, T., Wilhelm, R., Kalberla, P., \& Danly, L., 1996, ApJ, 473, 834

Wakker, B. P., van Woerden, H., Schwartz, U. J., Peletier, R. F., \& Douglas, N. G. 1996b, A\&A, 306, L25 